\title{
DARWIN EN LOS ALFARES: UN MARCO TEÓRICO EVOLUTIVO PARA ENTENDER LA PRODUCCIÓN CERÁMICA
}

Darwin in the pottery workshops: an evolutionary framework to understand the pottery production

5 María Coto-Sarmiento ${ }^{1}$

Palabras clave: producción cerámica - aprendizaje social - evolución cultural

Keywords: pottery production - social learning - Cultural Evolution

\section{RESUMEN}

La producción cerámica es una actividad cultural que permite trazar el modo en el que una comunidad transmite conocimientos. El resultado de explorar estos mecanismos de elaboración de un artefacto posibilita la reconstrucción de los procesos de producción de una parte de la estructura social que se refleja en el registro arqueológico. En este artículo se describe la importancia de un marco evolutivo para el estudio de las sociedades, enfocada en los contextos de producción cerámica. Se analizarán los principales mecanismos y formas de transmisión cultural que intervienen en el contexto de la elaboración de un artefacto, así como los nuevos retos con relación a los estudios evolutivos de artefactos dentro de una sociedad. Finalmente, se planteará una alternativa a los estudios historicistas clásicos con la combinación de un enfoque evolutivo que ofrece una serie de herramientas tanto teóricas como metodológicas al estudio de la producción cerámica.

\section{ABSTRACT}

Pottery production is a cultural and social activity that makes it possible to trace the way in which a community transmits practical knowledge. The result of exploring these mechanisms for the elaboration of an artifact enables the reconstruction of the production processes of a part of the social structure that is reflected in the archaeological record. This work describes the importance of an evolutionary framework in order to analyse the past societies, focused on the contexts of pottery production. In particular, the main mechanisms of cultural transmission that intervene in the context of the elaboration of an artifact will be analysed. In this work, a previous background in

1 PALAEOSILKROAD Project. University of Tübingen. 
archaeology will be described, as well as the new challenges in relation to the evolutionary studies of artifacts within a human society. Finally, an alternative to classical studies will be proposed with the combination of an evolutionary approach that offers a series of both theoretical and methodological tools to the study of pottery production in the past.

\section{UN ANÁLISIS DEL CAMBIO CULTURAL EN LA SOCIEDAD}

Desde nuestros ancestros hasta el ser humano actual, la cultura ha sido esencial para comprender los mecanismos del comportamiento humano a lo largo de nuestra historia. El término cultura se define como toda información socialmente transmitida y compartida a través de generaciones que afecta a la conducta y al desarrollo del ser humano (Richerson y Boyd, 2005; Boyd et al., 2011). La cultura será el resultado de un conocimiento adquirido y modificado a través del aprendizaje social y que puede repercutir en nuestro comportamiento de diferente manera (Mesoudi, 2011: 3). Ésta puede ser variada y estar envuelta en multitud de fenómenos como creencias, ideas o toma de decisiones, entre otros (Richerson y Boyd, 2005).

Las formas de interacción social entre personas que afectan a la conducta de un individuo se conoce como transmisión cultural y se relaciona con la idea de aplicar los preceptos biológicos al estudio del cambio cultural (Mesoudi, 2015). La existencia de una coevolución entre la cultura y la biología se conecta dentro de la Teoría de la Herencia dual (THD). La Teoría de la Herencia Dual se remonta a los escritos de Darwin donde se comparan los procesos biológicos con los culturales (Darwin, 1888). Las ideas en torno a esta teoría serán desarrolladas con posterioridad a partir de los años 60

50 donde tendrán especial repercusión una serie de publicaciones (Cavalli-Sforza y Feldman, 1981; Boyd y Richerson, 1985; Lumsden y Wilson, 2005; Boyd et al., 2011).

En el proceso de coevolución, el resultado de la combinación entre la evolución cultural y la biológica describirían acciones capaces de afectar al comportamiento de los individuos. El comportamiento del ser humano y la forma de adquirir conocimientos dependerá de los mecanismos culturales que cada sociedad haya adquirido, por lo que para entender el comportamiento de una persona no solo será necesario comprender los patrones biológicos, sino también los culturales (Shennan, 2008). En este caso, la cultura es vista como un ente esencial para la conducta del ser humano que puede transmitirse y replicarse al igual que nuestro sistema genético, aunque esta información transmitida no siempre la misma y variará dependiendo de los mecanismos que se usen para difundirla. 
Muchas son las cuestiones que existen alrededor del concepto cultura. Definir cultura plantea una serie de interrogantes con relación a cómo afectaría a las comunidades humanas. Una interesante descripción sobre el término cultura se encuentra en las categorías definidas por L. Binford (Binford, 1972: 117). Para Binford, la cultura puede definirse en base a ocho estamentos:

- La cultura se da en los seres vivos. La cultura se puede manifestar en diferentes aspectos que pueden definirse como ideas, creencias, comportamientos, etc.

- La cultura es continua. La cultura se transmite de generación en generación siendo continua en el tiempo y en el espacio.

- La cultura es transmitida entre generaciones. La cultura se transmite entre generaciones mediante el aprendizaje social que permite asimilar ideas de otros individuos, modificarlas y transmitirlas a través de generaciones.

-La cultura se comparte. La cultura permite la interacción entre individuos como, por ejemplo, la cooperación a la hora de fabricar un material.

- La cultura es acumulativa. Las ideas pueden ser adquiridas y modificadas por el individuo. La cultura se puede acumular para desencadenar nuevas ideas o modificar las antiguas. Estas ideas pueden modificarse a través de un proceso de recombinación para generar nuevas ideas.

- La cultura cambia. Puede cambiar constantemente, modificarse, etc. No permanece intacta en el tiempo.

- La cultura tiende a ser gradual pero no siempre. Los cambios con frecuencia pueden ser continuos tendiendo a la complejidad. Los cambios graduales pueden ser mayores o menores dependiendo del contexto. En algunos casos históricos, puede llegar a producirse una ruptura cultural a causa de acontecimientos como invasiones, guerras, etc.

\section{LA TRANSMISIÓN CULTURAL DEL APRENDIZAJE}

Son diversos los factores que entran dentro de este juego basado en la selección natural. En el caso de la cultura, uno de los modos de transmisión será por medio del aprendizaje social entre individuos. El aprendizaje social se entiende como el resultado de la enseñanza a través de interacciones entre seres vivos. 
90 Las formas de aprendizaje social pueden ser variadas dependiendo de los tipos de mecanismos de aprendizaje empleados, pudiendo ser por estímulos, donde el individuo aprende por reflejos tanto directos como indirectos, lo que quiere decir que el individuo es influenciado por el comportamiento de otro; emulación, donde el individuo trata de imitar a otro individuo; imitación donde el individuo observa, aprende y copia un modelo exacto de otro individuo y, por último, enseñanza, cuando un individuo enseña a otro individuo. Todos estos mecanismos dependerán directa o indirectamente de la supervisión de otro individuo. Si bien, no necesariamente se debe vincular un único mecanismo a procesos extremadamente complejos, puesto que variarán dependiendo de diversos factores (Lycett, 2015).

Así pues, dependiendo del tipo de transmisión que tenga lugar, los resultados podrían cambiar en el contexto y en el contenido. Al igual que la transmisión genética, es importante tener en cuenta que existen tres tipos de transmisión cultural definidas: vertical, horizontal y oblicua (Cavalli-Sforza y Feldman, 1981; Acerbi y Parisi, 2006) (ver fig. 1).

Fig. 1. Modos de transmisión cultural entre individuos: vertical donde la transmisión es entre parentescos; oblicua y horizontal donde no eiste ese parentesco. Imagen modificada y redibujada basada en Mesoudi (Mesoudi, 2019: 11).

\section{a) Transmisión vertical}

En cuanto a la transmisión vertical, el modelo de aprendizaje es el resultado de una transmisión entre parentescos, es decir, implica consanguinidad, a descendientes. Se trataría de un modo de transmisión parental especialmente vinculado a sociedades tradicionales de pequeña escala (CavalliSforza y Feldman, 1981; Acerbi y Parisi, 2006). En este modo de transmisión, la ratio de evolución resulta bastante conservativo y, por lo tanto, su evolución es lenta debido a que el contacto únicamente se hace por vía parental (Cavalli-Sforza y Feldman, 1981: 129). La variabilidad de este contacto sería alta, puesto que la transmisión se realiza dentro de un núcleo específico sin ninguna

115 conexión con otros individuos (Shennan, 2002).

\section{b) Transmisión horizontal}

Se trataría del modelo típico y más frecuente de transmisión cultural. Transmisión horizontal sería cuando el aprendizaje es transmitido entre individuos dentro de una misma generación de forma contemporánea y sin ningún parentesco. El modelo horizontal se encuentra estrechamente 
relacionado con la transmisión de ideas vinculadas a un sistema de intercambios y contactos continuados con otras culturas (Cavalli-Sforza y Feldman, 1981: 129). El contacto entre diversos tipos de individuos daría lugar a un ratio de evolución mucho más rápido que con cualquier otro tipo de transmisión, generando a posteriori una variabilidad menos pronunciada con respecto a la transmisión vertical debido a que las ideas se comparten de forma más rápida (Shennan, 2002).

\section{c) Transmisión oblicua}

A diferencia de la transmisión horizontal, en la transmisión oblicua el aprendizaje es transmitido de una generación anterior a otra más reciente, sin ningún tipo de parentesco entre ellas. También se produce de forma contemporánea. La transmisión de conocimientos suele darse únicamente por vía del aprendizaje o la imitación. Este tipo de intercambio de mecanismos de conocimiento es conocido en otras sociedades a través de estudios etnológicos como una forma de perpetuar el oficio y aprendizaje heredados (Epstein, 1998; Bowser y Patton, 2008).

La detección en el registro arqueológico de los diferentes modos de transmisión será esencial para explorar distintos mecanismos de aprendizaje social, así como para conocer el impacto que producen en la organización productiva de una comunidad.

\section{LA DEFINICIÓN DE EVOLUCIÓN CULTURAL: UN BREVE REPASO A SUS ANTECEDENTES}

Es necesario remontarse al siglo XIX para hablar de la idea de cultura como fiel representación de la evolución humana. Durante ese tiempo, el término evolución se relacionaba exclusivamente con la idea de progreso unilineal. Se entiende que existen sociedades que tienden a evolucionar unas más que otras y éstas son divididas en sociedades avanzadas cuando alcanzan una mayor complejidad, a diferencia de otras sociedades consideradas no civilizadas por su menor nivel de complejidad. Uno de los primeros pensadores que hizo referencia a la idea de progreso fue Spencer. En Principles of Sociology, Spencer recogió la teoría darwinista para esbozar su idea sobre el desarrollo de sociedades basadas en el concepto de complejidad social (Spencer, 1898). Para aquella época, existían diferentes tipos de sociedades que parten de distintas escalas desde la más simple, sin ningún modo de organización social, hasta la más compleja, reflejo de la sociedad industrial actual (Gordon Childe, 1984: 11).

La visión de sociedad compleja para la época se encontraba estrechamente relacionada con los ideales europeos de dominio y con la justificación de explotación hacia otros países "menos 
desarrollados” (Mesoudi, 2015: 37). Dentro de este pensamiento, la evolución es vista como una carrera de competición donde la idea de progreso es inevitable hasta llegar a la máxima escala social de complejidad relacionado con las sociedades europeas dominantes.

Con posterioridad, las ideas de Spencer fueron recogidas por el darwinismo social para desarrollar los postulados teóricos del darwinismo al estudio social. Este hecho hizo que surgieran las primeras tentativas de utilizar la teoría de selección natural de Darwin enfocada al análisis de las sociedades humanas. El darwinismo social vinculaba la evolución con la idea de supervivencia del más apto, considerando como más apto el más fuerte. De esta manera, las sociedades más avanzadas eran consideradas las más aptas dentro de un sistema competitivo por recursos.

La idea de progreso del darwinismo social sería posteriormente mal adaptada para justificar fines políticos o ideales racistas (Mesoudi, 2015: 40). Las sociedades más aptas justificarían así cualquier conquista como símbolo de progreso. De esta manera, el concepto de adaptación acabará siendo desvirtuado por completo llegando a usarse para justificar políticas colonialistas y racistas basadas en el dominio o conquista de unas sociedades sobre otras.

Dentro de este marco, la idea de evolución cultural estará más ligada a un concepto propiamente general, sin tener en cuenta la particularidad de cada sociedad y el porqué de su diversidad. Otro punto cuestionable será su enfoque exclusivamente progresista, considerando que cada sociedad debe alcanzar el estatus más alto de civilización, normalmente vinculado con el modelo europeo, aunque sin tener en cuenta otras particularidades o factores que podrían afectar a este proceso (Johnson, 2000: 117).

170 De manera simultánea, empiezan a tomar forma las ideas de Charles Darwin sobre el concepto evolución, suponiendo una verdadera revolución en los estudios evolutivos la publicación de su libro The Origin of Species en 1859. A diferencia de Spencer, Darwin no tiene en cuenta la idea de progreso asociada a mayor complejidad, siendo las especies capaces de coevolucionar, estancarse o extinguirse.

175 En uno de sus preceptos sobre variación, Darwin se basa en la observación biológica de que las especies no son inmutables, sino que experimentan variaciones de generación en generación dando lugar a nuevas especies resultantes de una serie de mutaciones genéticas que dependerán del contexto. 
Aunque no fue en The Origin of Species, sino en la publicación de The descent of man, and selection in relation to sex donde Darwin sintetiza lo que sería la posterior teoría de la evolución cultural. Darwin incluye el término de evolución cultural para explicar los paralelismos evolutivos que existen entre el lenguaje y la biología; esto es, la formación de diferentes lenguajes es producto del resultado de una serie de procesos graduales que habrían provocado cambios a través del tiempo al igual que las especies (Darwin, 1888: 59-60). La idea de una posible mutabilidad será aplicada al concepto de cultura: la cultura no es un ente estático, sino que va cambiando dependiendo de diferentes contextos (Mesoudi, 2011: 21).

El enfoque biológico se adoptaría para explicar el cambio cultural. En otras palabras, en la evolución cultural el conocimiento se adquiere y transmite por vía cultural y con un cierto propósito, mientras que en la biológica es por vía genética y puede ser resultado de mutaciones al azar, aunque hay ciertas excepciones (Mesoudi, 2011).

Las condiciones que afectan a los cambios que ocurren en la evolución biológica también podrían ser adaptadas para explicar la evolución cultural. Esto ha generado un interesante debate sobre si el proceso de cambio cultural podría ser descrito usando las teorías darwinistas.

Es necesario considerar que Darwin describe el proceso evolutivo biológico en base a tres condiciones (Darwin, 1859; Mesoudi, 2015). A partir de aquí, se discuten sobre si tales procesos darwinistas afectarían también al cambio cultural o no:

- Variación. Los organismos biológicos varían con el tiempo mediante la mutación genética y la recombinación de genes. La cultura también puede variar. Esto se refleja, por ejemplo, en que no existen las mismas religiones, opiniones políticas o similares artefactos en diferentes épocas. La variación cultural, al igual que la biológica, demuestra que se dan diferentes rasgos dentro de la cultura que pueden ser cuantificados y documentados con el estudio de la variabilidad de elementos.

- Competición. La competición es vista como una lucha por la existencia. Con frecuencia, la competición se relaciona con la limitación de recursos ante un crecimiento y en el que solo una parte podrá sobrevivir. En el caso de la cultura, la competición se extrapola al concepto de extinción, al igual que la extinción de especies debido a la falta de recursos. Existe multitud de documentación histórica y arqueológica que explica cómo determinadas tecnologías, artefactos e incluso lenguajes se han impuesto sobre otros, provocando la desaparición o adaptación por uno nuevo que se adapte mejor a las condiciones. Esto puede compararse al resultado obtenido producto 
210 de una competición entre ideas que provocará que un ente cultural predomine sobre otro. Mesoudi enfatiza en que esta competición se debe a una limitación en cuanto a recursos en la memoria de un individuo. Esto quiere decir que el individuo es únicamente capaz de memorizar una capacidad limitada de recursos, lo que hace que tenga que elegir entre unos u otros (Mesoudi, 2015: 31).

- Herencia. Los individuos heredan las características por parentesco. En el enfoque cultural, los rasgos también pueden ser transmitidos culturalmente entre parentescos. Es más probable que se adopten rasgos culturales como ideas, creencias, etc. entre individuos que compartan consanguinidad. La herencia cultural, al igual que los genes, puede modificarse con el tiempo a través de generaciones. Como ejemplo, el resultado de la elaboración de un artefacto puede deberse a una serie de modificaciones anteriores a través de recombinaciones para mejorarlo (Basalla, 1988).

El pensamiento darwinista se ha ido manteniendo durante décadas hasta la actualidad. El uso de esta teoría ha permitido además ser extrapolada al estudio de las sociedades humanas para explicar la existencia de diferentes rasgos culturales entre poblaciones que pueden variar en el tiempo y en el espacio.

225 No debe resultar extraño que similares mecanismos biológicos puedan encontrarse también en la cultura basados en los rasgos que determinan las características de un individuo como el genotipo y el fenotipo. En biología, se denomina genotipo a la información genética del organismo, mientras que fenotipo correspondería a los caracteres visibles de una especie (estatura, color de ojos, etc.). Este tipo de mecanismos también se manifiestan de una forma u otra en el caso de la cultura: el genotipo sería toda la información que se transmite, por ejemplo, a la hora de fabricar un artefacto, mientras que el fenotipo sería el propio artefacto (Neff, 1992: 141). Sin embargo, no hay un consenso claro entre investigadores sobre si el genotipo se podría dar estrictamente en la cultura, considerándose más adecuado hablar de replicadores culturales o memes (Dawkins, 1989; Gabora, 2004).

235 De acuerdo con esta premisa, las sociedades no serían entes estáticos, pudiendo variar progresivamente de acuerdo con diversos factores tanto endógenos como exógenos, dando lugar a generaciones cada vez más complejas o a colapsos. Aquí entraría el concepto de diversidad de especies entonces planteado por Darwin pero enfocado en la evolución cultural. De la misma forma que existen multitud de especies biológicas en la tierra, también existen abundantes vestigios que reflejan esta diversidad del ser humano (Basalla, 1988). 
Las ideas darwinistas tendrán una gran repercusión a nivel metodológico entre diferentes disciplinas o escuelas. A partir de los años 30 del siglo XX, un grupo de científicos, denominados a posteriori como neodarwinistas, añadieron el concepto de genes como motor del cambio evolutivo y la mutación genética para completar la teoría evolutiva de Darwin. De acuerdo con el neodarwinismo, los cambios serían producto de mutaciones que ayudarían a formar nuevas especies a diferencia del darwinismo, donde las transformaciones son el resultado de una acumulación de continúas variaciones que formarían nuevas especies. Las variaciones se acumularían en los genes y no en los individuos. Este argumento chocaría con el lamarckismo que considera que los rasgos adquiridos son heredados (Lyman y O’Brien, 1998: 625).

Durante este tiempo, se ha ido gestando un intenso debate sobre si algunas ideas de las teorías neodarwinistas podían ser incluidas o no en los estudios de evolución cultural, sobre todo por el absoluto rechazo al lamarckismo de los neodarwinistas. Algunos investigadores consideran que se trataría más de un problema de corte metodológico: en la evolución cultural puede ocurrir que la transmisión de ideas, conocimientos, etc. pueda ser el resultado de características adquiridas heredadas, tal y como ocurre en el lamarckismo (Mesoudi, 2015: 44).

Este problema y otros muchos relacionados con el uso de la teoría darwinista al estudio de la evolución cultural se intentarán solventar a partir del siglo XX, cuando se desarrollen las primeras herramientas cuantitativas con el propósito de modelar las diferencias que existen entre la evolución cultural y la biológica desde un enfoque evolutivo. Coincidiendo con el avance tecnológico, surgen

260 las primeras técnicas en forma de modelos formales matemáticos que permitirán poder reconstruir procesos evolutivos tanto a micro como a macro escala a lo largo del tiempo, destacando diversos trabajos en el área de las ciencias sociales (Cavalli-Sforza y Feldman, 1981; Boyd y Richerson, 1985; Shennan, 2002; Richerson y Boyd, 2005; Mesoudi, 2015). Este tipo de investigaciones se han enfocado principalmente en las dinámicas del cambio cultural con respecto a los artefactos arqueológicos a través del uso de una teoría evolutiva (Lycett, 2015).

El enfoque evolutivo para el estudio del comportamiento humano ha sido una premisa esencial para generar conocimiento, aunque su papel no ha estado lo suficientemente aceptado dentro de las ciencias humanas centradas en el estudio del pasado. La llegada de nuevas corrientes evolutivas a la arqueología, junto con el desarrollo y auge de nuevos modelos cuantitativos y de simulación, supuso un cambio radical en materia de entender los contextos culturales en base a un enfoque evolutivo. 


\section{ARQUEOLOGÍA Y EVOLUCIÓN CULTURAL}

Las manifestaciones culturales de una sociedad se ven con frecuencia reflejadas en el registro arqueológico a través de su cultura material. El hallazgo de diferentes artefactos permite conocer la existencia de una diversidad que no permanece intacta en el tiempo o en el espacio. La cultura material representa todo un abanico de información sobre creencias, patrones de conducta, ideas, lenguajes, producción, entre otras; de hecho, puede proporcionar información sobre el comportamiento del ser humano desde décadas y los cambios que va experimentando con el tiempo. No obstante, la palabra cultura ha comprendido a lo largo de la historia un extenso abanico de connotaciones y significados de todo tipo (Shennan, 2008; Mesoudi, 2011).

El interés por las culturas históricas se encuentra paralelamente vinculado con el surgimiento del coleccionismo. Aunque parece que no existe una fecha clara para los inicios del coleccionismo, se piensa que alcanzó su madurez durante la época absolutista en Europa (Hernández Hernández, 1992: 85).

En estos momentos se está gestando un interés en conocer la naturaleza de las civilizaciones y su pasado a través de la recolección de materiales arqueológicos desde diferentes regiones. Los inicios del coleccionismo se encuentran fuertemente ligados a una política colonialista basada en el expolio hacia otros países (Hernández Hernández, 1992). Como resultado de esta intensa política, surgiría el interés de crear un protocolo para albergar todas las piezas que se habían obtenido producto del coleccionismo privado o de diferentes campañas arqueológicas realizadas por todo el mundo. Así, la necesidad de buscar un espacio para guardar estas piezas supuso el inicio de lo que serían los primeros precedentes de los museos actuales (Hernández Hernández, 1994).

La construcción de los nuevos edificios museísticos permitió albergar diferentes colecciones estimulando a la par la curiosidad de los investigadores sobre el origen y significado de distintas sociedades. Como forma de ordenar las colecciones, se ideó una nueva metodología para clasificar los materiales arqueológicos basada en las clasificaciones taxonómicas que se venían practicando en otras disciplinas (Escacena et al., 2010; García Rivero, 2016b).

La práctica de la taxonomía abarca desde el siglo XVII, formalizándose a partir del siglo XVIII con la obra Systema Naturae escrita por Linneo. El mismo autor empleó el método de similitud como forma de clasificar especies y animales, aunque sin tener en cuenta su dinamismo evolutivo. Según García Rivero, la taxonomía se venía practicando desde el siglo XVII convirtiéndose en disciplina a 
partir del siglo XVIII, aunque el individuo siempre ha demostrado una tendencia a la clasificación por grupos (García Rivero, 2016b: 13).

305 Una de las múltiples razones se debió a que muchas piezas arqueológicas parecían bastante similares pero tenían ciertas particularidades que las hacían ser distintas entre ellas. El uso de la taxonomía permitió así poder ordenarlas, ya que se trataba de una clasificación basada en la similitud de artefactos. La taxonomía crearía una serie de agrupaciones de objetos y familias teniendo como único criterio la semejanza (García Rivero, 2012). De forma paralela, aparecen las

310 primeras clasificaciones históricas en relación con la ordenación de los materiales expuestos, generando así un interés en explicar los diferentes estadios culturales de las sociedades pasadas (Lane-Fox Pitt-Rivers y Myres, 1906). Las diferencias encontradas en la tecnología de los artefactos permitió a varios investigadores crear posibles clasificaciones, como la desarrollada por Thomsen usando los materiales depositados en el Museo de Antigüedades Nórdicas de Copenhague para establecer tres Edades históricas: Piedra, Bronce y Hierro y a la que sucederán otras clasificaciones más específicas hasta la actualidad (Gordon Childe, 1984). El interés por la taxonomía dará lugar a la creación futura de diferentes tipos de escuelas para establecer clasificaciones históricas basadas en aspectos sociales, cronológicos o tecnológicos que hoy en día se siguen utilizando (García Rivero, 2010).

Tradicionalmente, la arqueología se ha encargado de estudiar los procesos culturales que han permitido conocer y comprender los mecanismos por el cual diferentes comunidades evolucionan de una forma diferente a otras (Richerson y Boyd, 2005). No es de extrañar, dado que desde sus inicios la arqueología ha tratado de comprender los procesos de cambio en las sociedades pasadas y 325 presentes reflejados en la diversidad de su cultura material. Explicar las razones de estos cambios en la tecnología ha sido uno de los objetivos primordiales tanto para arqueólogos como historiadores como una forma esencial de conocer el pasado (Basalla, 1988).

\section{METODOLOGÍA EVOLUTIVA APLICADA AL ESTUDIO DE CONTEXTOS ARQUEOLÓGICOS}

330 Una de las técnicas más usadas en arqueología para estudiar diferencias en los artefactos sigue siendo la taxonomía. La taxonomía es una clasificación de tipo fenética donde se prioriza la similitud de los artefactos por su morfología, siendo los artefactos con rasgos más similares los más cercanos entre sí (García Rivero, 2012: 77). 
Un ejemplo de ello es la tendencia arraigada de utilizar la cerámica como indicador cronológico que sigue siendo esencial para fechar los niveles de ocupación a partir de los cambios relacionados con su morfologia. Como forma de detectar estos cambios, desde un principio se tendió a explicar este proceso a partir de clasificaciones basadas en variaciones morfométricas relacionadas con la similitud entre artefactos. Si bien, ha sido posible debido a que una buena parte de los artefactos cerámicos presentan pautas de evolución morfológicas susceptibles de ser reconocidas a simple vista y reconstruidas en un sentido diacrónico. La eficacia de este tipo de clasificaciones ha permitido su perdurabilidad como método a lo largo de los años a pesar de depender en cierta manera de la habilidad y percepción del ser humano (Eerkens y Bettinger, 2008).

Este método ha sido ampliamente utilizado en arqueología para establecer paralelismos con materiales y culturas por similitud, aunque con ciertos inconvenientes. Con frecuencia, el análisis taxonómico corresponde a clasificaciones basadas en el parecido de artefactos, pero sin ningún criterio evolutivo más que poseer una buena vista o similitudes en las formas.

Los artefactos también se clasifican en base a su complejidad. Esto quiere decir que la complejidad cultural se mide por la dificultad en elaborar un material atendiendo desde lo más antiguo (simple) hasta lo más nuevo (complejo) como marco cronológico. Esto puede tener cierta lógica si no fuese porque la taxonomía clásica puede carecer de un sentido multilineal. Normalmente, se fundamenta en modelos unilineales que parten de una única idea de evolución jerárquica, pero sin perspectiva evolutiva.

Por lo tanto, en el caso de la taxonomía, si se trata de un artefacto con una dificultad mayor en su elaboración es porque ha sido fabricado por una cultura con mayor complejidad. Con este criterio, las sociedades alcanzarían un mayor grado de complejidad en la elaboración y perfeccionamiento técnico de un artefacto. Por el contrario, no siempre es así cuando se aplica en mismo parámetro para las elaboraciones simples, con frecuencia relacionadas erróneamente con culturas que no han alcanzado un cierto grado de complejidad elevado.

Como forma de solventar este problema, surgieron nuevas disciplinas como la filogenética. La filogenética permite incluir el enfoque evolutivo necesario para comprender las dinámicas de cambio en los materiales arqueológicos, contemplando las variaciones basadas en la transmisión cultural y las relaciones evolutivas entre especies. De nuevo, Darwin sería el encargado de realizar los primeros esbozos mediante el dibujo de un árbol filogenético que representaría la evolución de las especies de forma más multilineal. 
Uno de los métodos más utilizados en la filogenética para el estudio de relaciones evolutivas entre organismos es la cladística (O’Brien et al., 2001). En arqueología, la cladística se ha planteado como una metodología alternativa y más completa a la taxonomía. En los últimos años, se ha empleado principalmente para el estudio de todo tipo de materiales tanto arqueológicos como actuales (O’Brien y Holland, 1990; Tehrani y Collard, 2002; Tëmkin y Eldredge, 2007; O’Brien et al., 2008; García Rivero, 2012; García Rivero y O’Brien, 2014).

Pese a que la cladística es ampliamente utilizada en otras disciplinas, una de las razones de su uso en arqueología ha sido la similitud que existe entre los datos biológicos y los culturales, en tanto que la cultura como los genes pueden ser transmitidos y modificados. De hecho, este método ofrece una herramienta eficaz para la reconstrucción morfológica de los artefactos basándose en los preceptos evolutivos (O’Brien et al., 2001). La cladística comparte con la taxonomía el principio de similitud con la diferencia de que ésta la hace por razones evolutivas creando una serie de secuencias que permiten reconstruir árboles filogenéticos. No obstante, el método cladístico no ha logrado tener el éxito esperado en la comunidad arqueológica. El uso que se le ha dado en arqueología sigue sin adaptarse por completo como sistema de clasificación. Esto podría deberse a varios factores relacionados con el abandono de los métodos científicos por parte del discurso postprocesualista en el sector arqueológico o a la limitada formación en competencias científicotécnicas del sector arqueológico.

Asimismo, existe un serie de interrogantes sobre si es posible reconstruir los procesos culturales 385 como los biológicos bajo un paraguas darwinista (O’Brien et al., 2008: 47). Esto podría explicarse en base a varias premisas. La primera es que el proceso de cambio entre los procesos culturales y los biológicos podría ser diferente, siendo mayoritariamente la ratio de cambio más rápido en los atributos culturales que en los genes (Shennan, 2008: 76). La segunda es que estos cambios no siempre son similares o comparables a los de otras especies. Esto se basa en el principio de la parsimonia que es cuando se precisan menos cambios evolutivos (O’Brien et al., 2016). El principio de parsimonia contempla que ante diferentes fenómenos en igualdad de condiciones, el proceso con menos asunciones será el más probable dentro de todos (también conocido como la Navaja de Ockham). Esto quiere decir que la parsimonia seleccionará la explicación más simple posible dentro de otras razones que suponga menos cambios evolutivos ante un fenómeno (Fitch, 1971; García Rivero, 2012). En el caso de la evolución cultural, la consideración de este principio como único proceso posible no siempre ocurre, observándose también eventos de determinada complejidad que resultarían complicados de explicar, sobre todo en el caso de los procesos históricos. 
A todo esto hay que añadir la complicación que existe en explicar la transmisión horizontal dentro de la evolución cultural. La transmisión horizontal se encuentra mucho más presente en los procesos culturales que en los biológicos, donde en esta última predomina en mayor medida la transmisión vertical, lo que no quiere decir que no sea posible detectar casos de transmisión horizontal en especies (Nunn et al., 2006; Currie et al., 2010; Garc1® Rivero, 2016a). De cualquier manera, existen complicaciones a la hora de entender tales procesos desde un punto de vista arqueológico al tratarse de diferentes modos de transmisión más directos que difícilmente pueden ser detectados en los contextos arqueológicos.

De forma acertada, aquellos desafíos con respecto al enfoque cultural han podido solventarse en parte gracias a la aplicación de nuevos modelos matemáticos y de simulación (Greenhill et al., 2009; Currie et al., 2010). En las últimas décadas, la eclosión de equipos multidisciplinares de trabajo y la llegada de nuevas metodologías de corte científico a la arqueología han dado lugar al surgimiento de nuevas alternativas que expliquen estos procesos culturales de cambio en los contextos arqueológicos. La creación de equipos multidisciplinares se ha materializado a través de la formación de proyectos que estudian los acontecimientos históricos usando metodologías cuantitativas innovadoras como la simulación computacional o el análisis estadístico, entre otros (Caro Saiz et al., 2013; Remesal Rodriguez et al., 2014).

415 El uso de herramientas cuantitativas y computacionales ha facilitado explorar diferentes fenómenos relacionados con esta casuıstica mediante el análisis de diversos mecanismos de interacción de las sociedades pasadas. En parte, el avance tecnológico en paralelo mediante la aplicación de esta metodología al campo de la arqueología ha posibilitado reducir costes y tiempo de trabajo de una forma más rápida y eficaz (Shennan, 2008: 81).

420 En arqueología, la mayor parte de los estudios de este tipo se han centrado mayoritariamente en explorar la variación en los artefactos desde una perspectiva evolutiva (Neiman, 1995; Lipo y Madsen, 2001; Lyman y O’Brien, 2000; Mesoudi y O’Brien, 2008a;b; de Voogt et al.,2013; Schillinger et al., 2016b). Los procesos de variación son tenidos en cuenta en la evolución cultural visto que la cultura es transmitida y compartida a través del tiempo llevando inevitablemente a cambios en los artefactos arqueológicos (Lycett, 2015: 7). Estos cambios en la variabilidad de los artefactos pueden ser cuantificables empíricamente mediante el análisis de las diferentes formas.

A pesar de ello, la llegada de esta metodología a la arqueología no ha sido un camino fácil, incluso a pesar de haberse asumido como parte de la disciplina y demostrado ser una herramienta eficaz para 
430 el análisis de la incertidumbre en los contextos arqueológicos. En mayor medida, la escasa formación en materias técnicas y científicas y la necesidad de usar mecanismos técnicos mucho más desarrollados han impedido que pueda asumirse por completo a la arqueología, a diferencia de otras disciplinas donde se ha adoptado esta metodología con buenos resultados (Rubio-Campillo, 2017: $52)$.

435 De cualquier manera, el enfoque que se propone actualmente permite explorar los procesos evolutivos internos que actúan en la elaboración de un material para conocer una parte del comportamiento social de las comunidades pasadas.

\section{¿EN QUÉ PIENSA UNA PERSONA ALFARERA? UN ENFOQUE EVOLUTIVO PARA EL ANÁLISIS DE LA PRODUCCIÓN CERÁMICA}

440 La cerámica sigue siendo uno de los marcadores más característicos para conocer las dinámicas económico-sociales en la sociedad, siendo uno de los elementos materiales con mayor presencia en los contextos arqueológicos y que mejor ha sobrevivido al paso del tiempo. De la misma manera que los contenedores actuales, su forma y volumen se encuentran estrechamente relacionados con el contenido, el lugar de fabricación, ası1como otras variables culturales, sociales y económicas que determinan la cantidad y calidad de un producto (Bevan, 2014). Por lo tanto, son elementos esenciales para entender ciertas dinámicas económicas porque reflejan una particularidad de la sociedad.

Las diferencias reflejadas en la diversidad del registro arqueológico facilita poder reconstruir una parte esencial de los mecanismos sociales del comportamiento humano. Además, permite ampliar el conocimiento sobre el modo de aprendizaje social que adquiere propiamente cada sociedad. Todo ello no será más que una fiel representación de una serie de decisiones culturales que se tomaron a la hora de fabricar un artefacto, decisiones que van desde la producción hasta el consumo.

Son estos fenómenos culturales los que determinarán la complejidad y diversidad de una comunidad (Miton y Charbonneau, 2018). En el caso de la cultura material en contextos arqueológicos, el tipo 455 de estrategias no será fácilmente detectable dado que éstas suelen ser flexibles o combinadas en el tiempo.

IDENTIFICANDO EL APRENDIZAJE SOCIAL EN LOS ARTEFACTOS CERÁMICOS 
El aprendizaje social relacionado con la fabricación de un artefacto se encuentra envuelto en una serie de ideas basadas en la propia experiencia aprendida por uno mismo o a través de otros individuos (Boyd et al., 2011). Esto quiere decir que puede darse desde diferentes formas: imitación u observación entre individuos, donde el individuo observa y aprende reproduciendo un modelo de otro individuo con o sin supervisión; o autodidacta, donde el individuo copia un modelo sin la supervisión de otro individuo. Esto último supondría un sobrecoste en relación con la transmisión de información dentro del grupo a causa de la constante reinvención de conceptos. En consecuencia, cada grupo desarrolla dinámicas de enseñanza para transmitir información a las nuevas generaciones (Boyd et al., 2011; Kendal et al., 2018).

Por lo tanto, si aplicamos tales conceptos al contexto de trabajo, es decir, a la fabricación de cerámica, se necesitará años de experiencia y práctica para poder perfeccionar el producto, siendo únicamente posible mediante la acumulación de aprendizaje que le permita finalmente perfeccionar su técnica para conseguir el producto adecuado. La adopción de tales técnicas tendrá lugar mediante la acumulación de conocimientos a lo largo de generaciones.

El resultado de toda interacción que implique una enseñanza generacional se conoce como cultura acumulativa (Kendal et al., 2018: 652). En este proceso se tiende a conservar las modificaciones anteriores que generen mayores ventajas hasta que surjan otras nuevas que permitan modificarlas $y$ mejorarlas (Laland y O’Brien, 2011) (ver fig. 2).

Fig. 2. Descripción de nicho cultural. Nicho cultural se entiende como la capacidad del ser humano de acumular información a través de generaciones para desarrollar herramientas, ideas, etc.

Así, las modificaciones adoptadas se mantienen en el tiempo con la posibilidad de replicar el modelo hasta conseguir mejoras que generen mayor eficiencia, diversidad o que sean más simples o complejas. De hecho, los avances tecnológicos pueden producirse como resultado de toda transmisión cultural a partir de la acumulación de multitud de mejoras con el tiempo. En cuanto a la cultura acumulativa, se ha intentando explicar las causas que provocarían tal fenómeno sin llegar a una conclusión concreta. Pese a ello, se discute que el origen de estos avances estaría ligado a una 485 serie de causas relacionadas con las habilidades; en otros casos, no existe ninguna motivación o causa aparente que pueda explicar este fenómeno (Derex et al., 2019). 
Los mecanismos de cambio en la variación de un artefacto dependerá de ciertas estrategias de información transmitidas a través del aprendizaje social. La forma de usar tales estrategias pueden ser tanto intencionales basadas en diferentes procesos; o aleatorias, basadas en el error de copia.

490 Las formas de aprendizaje en evolución cultural pueden darse en dos tipos de contextos dependiendo de las decisiones tomadas. Por un lado, pueden basarse en contextos donde existen algún tipo de sesgo (transmisión sesgada); por otro, en contextos aleatorios donde se no se da ningún tipo de sesgo premeditado (aleatoria).

495 Como su nombre indica, la transmisión sesgada implica que existe un cierto sesgo que conlleva a una preferencia entre distintas variantes culturales. Tal y como se muestra en el gráfico de la fig. 3, existen multitudes de estrategias sociales que obedecen a distintos contextos y contenidos. Estas estrategias con sesgo pueden clasificarse en dos tipos: las que dependen del contextos y las que dependen del contenido.

500 Fig. 3. Diferentes estrategias de aprendizaje social. Gráfico basado en los anteriores trabajos correspondientes a (Henrich y McElreath, 2003; Lycett, 2015; Kendal et al., 2018) con algunas modificaciones posteriores

\section{Contenido o sesgo de Contenido}

505 Los sesgos de contenido son también denominados sesgos directos o de transmisión sesgada. Son los sesgos basados en preferencias sociales por rasgos culturales existentes. Esto se puede relacionar con la naturaleza de la información contenida. En este caso, los sesgos se pueden basar tanto en decisiones emocionales o culturales como en la funcionalidad de un producto. Un posible ejemplo se encuentra en la elaboración de un artefacto cerámico por el tamaño (sesgo funcional).

\section{Contexto o sesgo de Contexto}

Se trata de otro sesgo directo. Se basa en las decisiones que usan el contexto para determinar la variante cultural tomada. Se dividen en dos: sesgos basado en modelos y sesgos basados en la frecuencia.

a) Sesgos basados en modelos 
515 Corresponde a las estrategias o modelos culturales que el individuo imita. La imitación dependerá de diferentes factores basados en el prestigio, éxito, similitud o en características sociales (edad, sexo, etc.).

\section{b) Sesgos basados en la frecuencia (conformismo/anticonformismo)}

Son sesgos que se fundamentan en las elecciones de una variante cultural en relación con la

520 frecuencia en la que se observa en la sociedad; o en el tipo de frecuencia a la hora de desarrollar una estrategia, siendo más común adaptarse a una estrategia conformista (sesgo de conformismo) donde los individuos copiarán a la mayoría pensando que es la más efectiva. Este sesgo es bastante común en los comportamientos individuales donde influyen las decisiones tomadas por la mayoría (Boyd y Richerson, 1985; Mesoudi y O’Brien, 2009; Kendal et al., 2018). Lo contrario sería el sesgo anticonformista, donde el individuo copia los rasgos más raros y menos comunes adoptados por la población.

Los cambios también pueden producirse de forma aleatoria sin ningún tipo de sesgo destacable. Este proceso se conoce como deriva cultural y puede darse sobre todo cuando un individuo fabrica un material pero comete un error no deliberado (Eerkens y Lipo, 2005). Estos cambios se generan sin una motivación directa aparente y son denominados como aleatorios para diferenciarse de los que contienen sesgos. Así, los rasgos culturales van cambiando progresivamente de manera aleatoria, siendo el resultado de mutaciones consecuencia de posibles pequeños errores producidos que se van transmitiendo con el tiempo (Lipo y Madsen, 2001; Bentley et al., 2004; Eerkens y Lipo, 2005; Gandon et al., 2014).

Los pequeños errores en la fabricación de un producto pueden ser resultado de diversos motivos no deliberados, entre los que se encuentran la falta de información u observación inadecuada al replicar un modelo (mecanismo cognitivo) o condiciones físicas o ambientales que dan lugar a una serie de variaciones en el material resultante (Lycett, 2015). En el caso de errores no deliberados, un ejemplo puede ser cuando percibimos una pieza que tenemos que replicar más pequeña o grande de lo que realmente es o cuando se trabaja con diferentes materiales para fabricar un mismo artefacto.

Los estudios experimentales han sido útiles a la hora de detectar y comprender esta estrategia de comportamiento. De hecho, existen investigaciones realizadas con individuos para conocer la variación que puede existir en un material cuando es imitado dependiendo de diferentes contextos 545 (Schillinger et al., 2016a). 
Esta acumulación de errores puede incluso generar una nueva pieza o mantener la misma pieza con ciertas innovaciones inducidas por el mismo error. Si bien, se desconoce si puede darse como un único mecanismo o combinado con varios a la vez que contengan sesgos (Eerkens y Lipo, 2005: 317). Esto hace que sea difícil poder detectar cambios en un material arqueológico si no se usan las herramientas adecuadas, e incluso que algo deliberado sea visto como aleatorio.

Las diferencias en las estrategias de aprendizaje también podrían verse afectadas por diversos factores, entre ellos el espacial. En genética, hay una tendencia a que las poblaciones más cercanas entre sí tiendan a compartir mayores rasgos genéticos que las poblaciones que están más alejadas. Este factor es conocido como aislamiento por distancia donde la similitud o disimilitud de los atributos genéticos se asocia con la frecuencia geográfica, pese a que no siempre se cumple la norma en evolución cultural (Björklund et al., 2010; Shennan et al., 2015).

El proceso de aislamiento por distancia se usa para el análisis de dispersión de poblaciones, detección de enfermedades genéticas asociadas al aislamiento poblacional o migraciones, entre otros. Este fenómeno explica que la probabilidad de similitud entre dos comunidades dependerá de 560 la proximidad geográfica, siendo mayor la similitud cuando la proximidad es menor. De igual forma, se asocia con la primera ley de Tobler en geografía que afirma que todo está relacionado entre sí, pero las cosas más cercanas están más relacionadas (Tobler, 1970).

En arqueología, este fenómeno se ha enfocado en el estudio de similitudes morfométricas en artefactos para medir la probabilidad de compartir rasgos en la producción dependiendo de la distancia espacial (Aguilera Martín, 1998; Li et al., 2014; Coto-Sarmiento et al., 2018). Es decir, la probabilidad de compartir similares atributos en los materiales producidos por comunidades cercanas sería mucho más alta que en comunidades más alejadas. Este hecho puede influir también en los modos de transmisión cultural; esto es, si la transmisión vertical u oblicua fuese la predominante en este proceso, los artefactos fabricados en lugares más cercanos podrían compartir 570 rasgos más similares que los artefactos fabricados en sitios totalmente dispares. Si por el contrario, hubiese un predominio de la transmisión horizontal desde un principio, la producción seria la misma independientemente de la distancia geográfica debido a una progresiva homogeneización en la producción resultado de continuos contactos entre individuos que comparten las mismas técnicas de fabricación.

575 Los mecanismos de transmisión cultural también posibilitan analizar otros fenómenos como la mecánica de las innovaciones o estandarizaciones en los artefactos. En el caso de las innovaciones, 
la transmisión cultural podría afectar incluso a la optimización y a la comprensión de los sistemas que producen mejoras en los artefactos (Derex et al., 2019). En función del modo de transmisión, resultará más complicado la aceptación de cambios cuando existe una mayor presencia de transmisión vertical que de horizontal. Esto quiere decir que será más difícil innovar en la creación de un artefacto dentro de un seno familiar donde tradicionalmente se usa la misma técnica que, por el contrario, compartiendo técnicas con diferentes grupos de individuos que permitan aportar nuevas ideas (transmisión horizontal). En este último caso, la aceptación de un modelo innovador será más frecuente y fácil dado que el individuo está más expuesto a estímulos exteriores (Shennan, 585 2008: 51).

En cuanto a los procesos de estandarización, se relacionan con la existencia de mecanismos de alta fidelidad en los modos de transmisión para preservar un artefacto, aunque no siempre se produce. La estabilidad en la fabricación de los artefactos también podría deberse a otras condiciones relacionadas con procesos de convergencia, aún más cuando existe cierto sesgo que haga que se 590 produzca (Acerbi et al., 2019).

Todos estos mecanismos culturales ayudarán a explorar los procesos de transmisión asociados a la producción anfórica a través de una combinación de análisis empírico y exploración teórica.

\section{EL ANÁLISIS DE ARTEFACTOS CERÁMICOS COMO INDICADOR CULTURAL}

Dentro del marco de la evolución cultural, la mayor parte de estos trabajos se han centrado en el

595 estudio del comportamiento de diferentes sociedades humanas usando los materiales arqueológicos como su fiel reflejo. En particular, uno de los materiales más frecuentes encontrados en el registro arqueológico son los artefactos cerámicos. Los materiales cerámicos permiten conocer patrones de organización, producción y consumo de una sociedad. En cada yacimiento se pueden localizar una variedad ingente de diferentes tipos de cerámicas que podrían variar de forma temporal a pesar de pertenecer incluso a una misma tipología.

Una buena parte de los estudios de artefactos cerámicos se han centrado en explicar la razón de los cambios que se producen en su morfología mediante el análisis taxonómico basado en la similitud de las formas. Otro enfoque importante al estudio de artefactos cerámicos ha sido el evolutivo. Como se explicó anteriormente, en los últimos años, el enfoque evolutivo en arqueología ha estado 605 ligado al análisis de patrones de variación en distintos artefactos arqueológicos, usando una metodología más cuantitativa o de simulación como alternativa a los estudios de taxonomía clásica 
(Eerkens y Lipo, 2007; Mesoudi y O’Brien, 2008b; Li et al., 2014; Baldi y Roux, 2016). El propósito de buena parte de ellos ha sido principalmente examinar cómo los artefactos cerámicos fueron creados, transmitidos o eliminados a través del tiempo y las razones que evidenciaron tales cambios.

Una gran parte de estos estudios han abarcado los artefactos cerámicos hechos a mano (O’Brien y Holland, 1990; Neiman, 1995; Shennan y Wilkinson, 2001; Eerkens y Bettinger, 2008; Steele et al., 2010). La mayoría de los estudios versan sobre los procesos de evolución relacionados con la variación estilística o funcional. Con respecto a los estudios estilísticos, una explicación a este interés reside en que el tipo de decoración puede determinar el grado de complejidad de un artefacto cerámico basándose en la asunción de que los estilos más complejos podrían ser los más actuales (O’Brien y Holland, 1990: 52). En otros casos, la variabilidad en los artefactos ha sido analizada mediante el uso de dos tipos de metodologías: los análisis etnográficos o de laboratorio y el uso de modelos cuantitativos o matemáticos.

Los análisis etnográficos o de laboratorio se basan en la observación de los procesos de fabricación de un artefacto cerámico de una determinada cultura con la idea de extrapolarlo a los estudios del pasado. En ese sentido, se mide tanto la variación del artefacto cerámico como el modelo de transmisión en relación con las diferentes formas de producción (Bowser y Patton, 2008; Gandon et. al., 2014; Schillinger et al., 2016b; Gandon et. al., 2018). Esta idea resulta también útil a la hora de aplicar modelos estadísticos a los estudios cerámicos centrados en el análisis de la variabilidad tanto de la morfología como del estilo (Bentley et. al., 2004; Steele et al., 2010; Roux, 2015; Baldi y Roux, 2016; Coto-Sarmiento et al., 2018; Gandon et. al., 2018).

En general, los estudios evolutivos en cerámica raramente han sido aplicados en producciones mucho más industrializadas con la idea de comprender elaboraciones cerámicas mucho más estandarizadas. Afortunadamente, existen casos excepcionales que han tenido lugar en los últimos años, siendo posible gracias a la combinación entre diferentes disciplinas, aunque siguen siendo insuficientes en comparación con otras disciplinas (Aguilera, 1998; Epstein, 1998; Gandon et. al., 635 2014; Roux, 2015; Rubio-Campillo et al., 2017; Coto-Sarmiento et al., 2018; Rubio-Campillo et al., 2018).

El uso de una metodología cuantitativa y un enfoque evolutivo ha posibilitado conocer en mayor medida los procesos de cambio que experimentan las producciones cerámicas y tratar de explicar 
fenómenos políticos y sociales que afectan a la sociedad. Una futura combinación con la taxonomía 640 clásica puede ser efectiva a la hora de generar unos resultados mucho más científicos y menos descriptivos. En un futuro, el surgimiento de nuevas herramientas vinculadas al avance tecnológico en la disciplina y el auge de proyectos cada vez más interdisciplinares se convertirán en un soporte esencial para detectar diferentes fenómenos entre distintas producciones que son inapreciables en los contextos arqueológicos, así como una forma de analizar diversos mecanismos de interacción

645 en las sociedades pasadas.

\section{AGRADECIMIENTOS}

Este trabajo ha sido financiado por la European Research Council (ERC) bajo la European Union's Horizon 2020 research and innovation programme (grant agreement $n^{\circ}$ 714842; PALAEOSILKROAD project). 